Faculdade

de Ciências Econômicas UFRGS
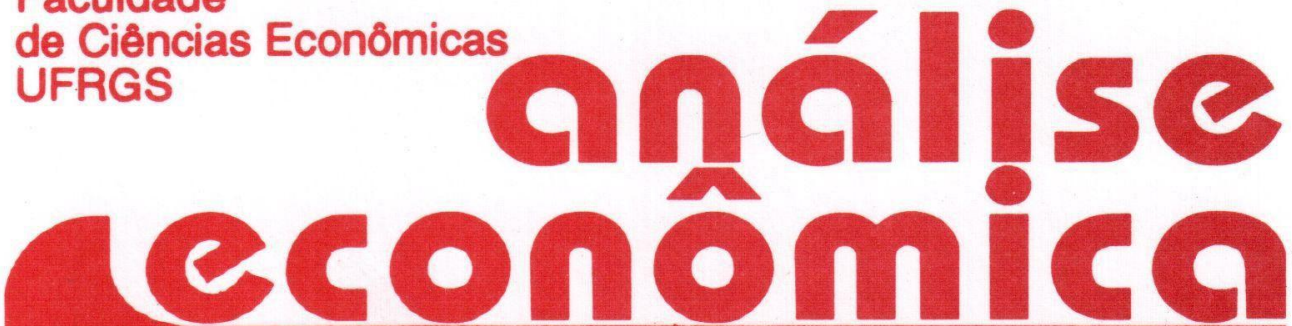

- A TEORIA NEOCLÁSSICA (PURA) E A TEORIA NEO-AUSTRIACA FRENTE AO LEGADO CARTESIANO Eleutério F. S. Prado

- MEdidas de concentração INDUSTRIAL: UMA RESENHA Marcelo Resende

- SISTEMA TRIBUTÁRIO E IMPOSTO ÚNICO SOBRE TRANSAÇÕES Ricardo Letizia Garcia

- ECONOMIA DO NORDESTE: TENDÊNCIAS DAS ÁREAS DINÂMICAS Policarpo Lima

- custos e Benefícios DA INTEGRAÇÃO DO GRUPO ANDINO Marco Antônio Montoya

- Pós-graduAÇÃo EM ECONOMIa NOS ESTADOS UNIDOS DA AMÉRICA Egon Roque Fröhlich




UNIVERSIDADE FEDERAL DO RIO GRANDE DO SUL

Reitor Prof Hélgio Henrique Casses Trindade

FACULDADE DE CIÊNCIAS ECONÓMICAS

Diretor Prof Pedro Cézar Dutra Fonseca

CENTRO DE ESTUDOS E PEQUISAS ECONÔMICAS

Diretor Prof. Paulo Alexandre Spohr

DEPARTAMENTO DE CIÉNCIAS ECONÓMICAS

Chefe: Prof Achyles Barcelos da Costa

CURSO DE PÓS-GRADUAÇÃO EM ECONOMIA

Coordenador Prof Nali de Jesus de Souza

CURSO DE PÓS-GRADUAÇÃO EM ECONOMIA RURAL

Coordenador. Prof Atos Freitas Grawunder

CONSELHO EDITORIAL: Achyles Barcelos da Costa, Aray Miguel Feldens, Atos Freitas Grawunder, Carlos Augusto Crusius, Fernando Ferrari Filho, Juvir Luiz Mattuella, Marcelo Savino Portugal, Maria Imilda da Costa e Silva, Nali de Jesus de Souza, Nuno R. L. de Figueiredo Pinto, Otília Beatriz K. Carrion, Paulo Alexandre Spohr, Pedro Cezar Dutra Fonseca, Roberto Camps Moraes, Valter José Stülp, David Garlow (Wharton Econometrics Forecasts Association, E.U.A.), Edgar Augusto Lanzer (UFSC), Eleutério Prado (USP), Fernando de Holanda Barbosa (FGV/RJ), Gustavo Franco (PUC/RJ), João Rogério Sanson (UFSC), Joaquim Pinto de Andrade (UnB), Juan H. Moldau (USP), Werner Baer (Univ. de Illinois, E.U.A.).

COMISSÃO EDITORIAL: Atos Freitas Grawunder, Pedro Cezar Dutra Fonseca, Marcelo Savino Portugal, Roberto Camps Moraes.

EDITOR: Nali de Jesus de Souza

SECRETARIA Cláudia Porto Silveira, Jeferson Luis Bittencourt e Vanete Ricachescki (revisão de textos)

FUNDADOR: Prof Antônio Carlos Santos Rosa

Os materiais publicados na revista Análise Econômica são da exclusiva responsabilidade dos autores. É permitida a reprodução total ou parcial dos trabalhos, desde que seia citada a fonte. Aceita-se permuta com revistas congêneres. Aceitam-se, também, livros para divulgação, elaboração de resenhas e recensōes. Toda correspondência, material para publicação (vide normas na terceira capa), assinaturas e permutas devem ser dirigidos ao seguinte destinatário:

PROF. NALI DE JESUS DE SOUZA

Revista Análise Econômica

Av. João Pessoa, 52

CEP 90040-000 PORTO ALEGRE - RS, BRASIL

E-MAIL: NALI@VORTEXUFRGS.BR

Telefones: (051) 316-3348 e 316-3440

Fax: (051) 225-1067 


\section{MEDIDAS DE CONCENTRAÇÃO INDUSTRIAL: UMA RESENHA}

Marcelo Resende

\section{SINOPSE}

Este trabalho apresenta em detalhes algumas medidas de concentração industrial, quais sejam, as razōes de concentração, os indices de Herfindahl e de entropia de Theil. Adicionalmente discute-se as vantagens dessas medidas, umas em relação às demais.

\section{INTRODUÇÃO}

Uma prática recorrente em estudos de organização industrial consiste na aproximação da estrutura de mercado por alguma medida de concentração. Cabe ressaltar, contudo, a pouca atenção dispensada à discussão acerca da adequação dos indices de concentração de uso corrente. Com efeito, grande parte dos estudos sobre concentração industrial fazem uso de medidas de concentração algo toscas. Assim sendo, o presente trabalho pretende preencher uma lacuna através da discussão detalhada de algumas medidas de concentração de uso freqüente na literatura

Uma primeira questão de interesse refere-se à possibilidade da estrutura de mercado ser aproximada por indices de concentração. O recurso à literatura de organização industrial não nos fornece, contudo, uma definição simples e não ambigua do que seria uma estrutura, mas sim uma coleção de características relativas aos lados da demanda e da oferta do bem ou serviço em questão. Nesse sentido, convém apresentar a definição proposta por Bain (1968). Segundo tal autor, as seguintes caracteristicas descrevem uma estrutura de mercado.

a) o grau de concentração descrito pelo número e distribuição de tamanho dos vendedores du mercado; a (a)];

b) o grau de concentração relativa aos compradores [definido de forma análoga

c) o grau de diferenciação do produto;

d) as condições de entrada no mercado (refere-se à existência de barreiras à eritrada).

"Professor Assistente do Departamento de Economia da UFRJ.

\begin{tabular}{|c|l|}
\hline $\begin{array}{c}\text { Cód. AEA } \\
610\end{array}$ & $\begin{array}{l}\text { Palavras-chave: organização industrial, } \\
\text { concentraçãoindustrial, medidas de concentração. }\end{array}$ \\
\hline
\end{tabular}


Vale destacar que os itens de tal lista não são mutuamente excludentes; em particular, o item (c) é um caso particular do item (d), no qual se tem barreiras a entrada relativas às preferências dos consumidores.

Como se depreende do anteriormente exposto, a estrutura de mercado é multidimensional o que torna sua mensuração uma questão controversa; tipicamente os dados disponiveis acabam por induzir ao cálculo de medidas relativas à oferta, as quais se acredita, denotariam de forma sintética o poder de mercado das firmas de uma dada indústria. Esquematicamente, podemos definir três etapas no desenvolvimento de medidas de estrutura de mercado:

a) o grau de concentração nas vendas descrito pelo número e distribuição de tamanho dos vendedores no mercado (ver Adelman 1961);

b) o grau de concentração nas vendas medidas em termos de participação das maiores firmas no mercado;

c) a intensidade da concentração medida em termos de um indice que considere todas as firmas que atuem em um dado mercado.

Passa-se assim de uma etapa na qual os estudos praticamente se limitam à contagem de firmas para chegar-se finalmente à utilização de índices que consideram, segundo algum esquema de ponderação, a participação de cada firma no mercado. De todo o modo, o que deve ficar claro, desde logo, é que ao utilizarmos índices de concentração, estaremos tentando resumir em um único indicador um conceito com múltiplas dimensões, o que indica a necessidade de análises complementares ao cálculo de indices de concentração entre si.

Uma primeira caracterização dos indices de concentração se refere à utilização; nesse sentido podemos classificá-los em dois grupos, a saber:

a) Parciais

b) Sumários

O primeiro grupo faz uso de apenas parte dos dados referentes à totalidade das firmas em questão, ao passo que os índices referentes ao segundo grupo consideram toda a informação da população amostral e não apenas as maiores firmas. Dentre as medidas parciais, destacam-se as chamadas razões de concentração ("concetration ratios"), enquanto que na categoria de medidas sumárias destacamos os indices de Herfindahl e o de entrnpia de Theil. ${ }^{1}$

O presente trabalho está organizado da seguinte forma: a segunda seção discute as chamadas razões de concentração, ao passc que a terceira e quarta seçōes discutem os indices de Herfindahl e de entropia de Theil, respectivamente. A quinta e última seção trata da escolha entre medidas de concentração alternativas.

\section{RAZÕES DE CONCENTRAÇÃO}

1 Essa medida é por vezes denominada de indices de Hirschman-Herfindahl, no entanto o indice de Hirschman foi formulado anteriormente ao indice de Herfindahl e sua fórmula é diferente:

$$
H i=\left(\sum_{i=1}^{n} P_{i}^{2}\right)^{1 / 2}
$$


Esses índices se estabelecem a partir da ordenação de forma decrescente da variável estudada. Isso posto, considera-se a participação das maiores firmas no total, ou seja, a razão das $m$ maiores firmas em um mercado com $n$ firmas seria definida como:

$$
C R_{m}=\frac{\sum_{i=1}^{m} X_{i}}{\sum_{i=1}^{n} X_{i}}=\sum_{i=1}^{m} p_{i}
$$

Onde $X$, representa a variável de interesse, e $p_{i}$ indica a parcela de mercado da i-ésima firma no total da variável.

Tipicamente, se trabalha com a participação das quatros ou oitos maiores $\left(\mathrm{CR}_{4} \mathrm{e}\right.$ $\mathrm{CR}_{8}$, respectivamente). Em que pese a facilidade de cálculo desse indice, existem diversas criticas ao seu uso, que enumeramos abaixo:

a) as $m$ maiores firmas em um período considerado podem não ser as mesmas em outros períodos;

b) desconsidera a concentração relativa entre firmas, seja dentro do grupo das $m$ maiores firmas ou no grupo das demais. Com efeito, fusões que ocorram dentro dos grupos das $n-m$ firmas não serão captadas em termos de um aumento do valor desse indice, tampouco serão consideradas mudanças na participaçāo relativa de cada firma pertencente ao grupo das $m$ maiores.

A consideração das deficiências supracitadas apontam na direção da utilização de medidas sumárias que ultrapassem tais dificuldades. Duas dessas medidas são apresentadas a seguir.

\section{INDICE DE HERFINDAHL (H)}

Tal índice é definido por

$$
H=\sum_{i=1}^{n} P_{i}^{2}
$$

Escrevendo essa fórmula de uma forma alternativa temos:

$$
H=\sum_{i=1}^{n} P_{i} W_{i}
$$

Assim procedendo, as parcelas de mercado de cada firma transformam-se nas ponderaçōes $W_{i}$, ou seja, $W_{i}=P_{i}$. Deve-se ressaltar que ao se elevar ao quadrado cada parcela $\mathrm{P}_{\mathrm{i}}$ estaremos atribuindo um peso maior às parcelas relativamente maiores.

O limite superior desse índice é igual a um, ocorrendo na situação de monopólio, pois nesse caso $p_{i}=i$ para algum i e $p_{j}=0$ para todo $j \neq i$.

$O$ valor minimo desse indice pode ser obtido resolvendo-se :

$$
H=\sum_{i=1}^{n} P_{i}^{2} \text { sujeito a } \sum_{i=1}^{n} P_{i}=1
$$

Assim fazendo o lagrangeano temos: 


$$
L=\sum_{i=1}^{n} P_{i}^{2}-\lambda\left(\sum_{i=1}^{n} P_{i}-1\right)
$$

Igualando-se a derivada parcial de $L$ com respeito a $P_{\mathrm{i}}$ a zero, tem-se:

$$
\frac{\partial L}{\partial P_{i}}=2 P_{i}-I=0
$$

Chega-se ao valor de $P_{i}$ igual a $\lambda / 2$; mas pela restrição temos que:

$\sum_{i=1}^{n} P_{i}=1$ logo, por substituição, tem-se que $\lambda=2 / n$, que substituido em (i) nos dá:

$$
2 p_{i}-2 / n=0 \quad p_{i}=1 / n
$$
logo:

Substituindo esse valor de $p_{i}$ na fórmula de $H$, temos que seu valor mínimo é $1 / n$,

$$
1 / n \leq H \leq 1
$$

Podemos notar que, à medida que o número de firmas aumenta, o limite inferior do indice de Herfindahl diminuirá, quando o número de firmas tende ao infinito, claramente o valor do índice tende para zero.

Como já fora afirmado anteriormente, o indice de Herfindahl capta fusões em termos de um aumento em seu valor. Consideremos uma situação inicial em que existem separadamente as firmas 1 e 2 , nesse caso o índice para a indústria seria:

$$
H_{0}=p_{1}^{2}+p_{2}^{2}+\sum_{1=3}^{n} p_{1}^{2}
$$

Num segundo momento, com a fusão das firmas 1 e 2 , o índice se modificará, admitindo que a participação da nova firma no mercado seja $p_{1}+p_{2}$, teríamos:

$$
H_{1}=\left(p_{1}+p_{2}\right)^{2}+\sum_{i=3}^{n} p_{i}^{2}=p_{1}^{2}+p_{2}^{2}+2 p_{1} p_{2}+\sum_{i=3}^{n} p_{1}^{2}=H_{0}+2 p_{1} p_{2}
$$

Desse modo, $\mathrm{H}_{\mathrm{i}} \geq \mathrm{H}_{0}$

Adicionalmente, é interessante observarmos que, a partir de tal indice, pode-se definir o número equivalente de firmas de igual tamanho que levaria ao mesmo indice $\mathrm{H}$. Esse número $\dot{e}$, de fato, o inverso desse indice. ${ }^{2}$

Admitindo-se que existam $n$ firmas de igual tamanho, isso significa dizer que $p_{1}=1 / n$ para todo i. Assim teremos:

$$
H=\sum_{i=1}^{n}(1 / n)^{2}=N .1 / N^{2}=1 / N
$$

Logo a proposição feita acima está provada.

Comó $\mathrm{H}$ está compreendido entre $1 / \mathrm{n}$ e 1 , temos que o número equivalente de

${ }^{2}$ Pretende-se, com tal medida, ter-se um indicador mais intuitivo do grau de competitividade do setor considerado. 
firmas de igual tamanho $\left(n_{0}\right)$ está compreendido no seguinte intervalo:

$$
i \leq n_{0} \leq n
$$

Uma limitação do índice $H$ é de que, à medida que $n$ muda, o limite inferior ( $1 / n)$ também se modifica, de tal sorte que comparaçōes intertemporais ficam prejudicadas. Assim fazem-se necessários ajustes nos indices, quando o número de firmas ao longo do período estudado estiver variando. ${ }^{3}$

Podemos ajustar o índice de $\mathrm{H}$ com respeito a amplitude de variação, definindo:

$$
H^{\prime}=\frac{n}{n-1} H \quad \text { para } n>1
$$

Nesse caso, os limites inferior e superior passariam a ser $1 /(n-1)$ e $n /(n-1)$ respectivamente. Temos, pois, que os valores extremos se modificam em função de $\mathrm{n}$; no entanto, a amplitude da variação é constante e igual à unidade

$$
\frac{n}{n-1}-\frac{1}{n-1}=1
$$

Podemos ir mais longe, sugerindo um ajuste que torne não só a amplitude de variação inciependente de $n$, como também os valores limites do indice, utilizando a seguinte fórmula:

$$
H^{\prime \prime}=\frac{1}{n-1}[\mathrm{nH}-1] \quad \text { para } n>1
$$

Para se obter os valores extremos de $\mathrm{H}^{\prime}$ e $\mathrm{H}^{\prime \prime}$, basta substituir os valores extremos de $H$ (que são $1 / n$ e 1) nas fórmulas ajustadas. Assim procedendo, ficará claro que os limites inferior e superior de $\mathrm{H}^{\prime \prime}$ são 0 e 1, respectivamente, e portanto são independentes de $\mathrm{n}$. Isso posto, podemos considerar "H" como a medida mais conveniente para comparações intertemporais.

\section{4. ÍNDICE DE ENTROPIA DE THEIL (ET)}

Esse índice surgiu no âmbito da teoria da informação e sua aplicação ocorreu em estudos de organização industrial seria sugerida por Theil (1967) e por Finkelstein \& Friedberg (1967)

Considere-se que a possibilidade, a priori, de ocorrência ùe um dado evento $A$ seja $\mathrm{p}$. Se posteriormente uma mensagem confirma a ocorrência de tal evento, o grau de surpresa emergente variará na direção contrária de $p$. Assim sendo, podese afirmar que o conteúdo de informação de uma mensagem (doravante denominado $h(p)$ ) guarda relação inversa com $p$. Dentre as possiveis funções decrescente que poderiam ser escolhidas, Theil escolhe a função logaritmo (multiplicada por -1), dada sua propriedade de aditividade, assim temos:

$$
h(p)=-\ln p
$$

Podemos generalizar o raciocínio anterior para $n$ eventos, $A_{1}, \ldots, A_{n}$, com probabilidades, $p_{i}, \ldots, p_{n}$. Tais probabilidade somam 1 ; uma vez que se sabe que um dos eventos ocorrerá. Se o evento $A_{1}$ ocorrer, a informação recebida será $h\left(p_{i}\right)=\ln p_{i}$

${ }^{3}$ A discussão que se segue se ampara em Srivastava \& Aggarwal (1979). 
como já foi explicado. Náo sabemos, a priori, qual o evento $A_{1}$ ocorrerá, mas sabemos que a probabilidade de ocorrência de $A_{1}$ é $p_{1}$. Assim a informaçáo recebida é $h\left(p_{i}\right)$ com probabilidade $p_{i,}$ tal que a informação esperada é:

$$
E T=\sum_{i=1}^{n} p_{i} h\left(p_{i}\right)=\sum_{i=1}^{n} p_{i} \ln 1 / p_{i}
$$

Nesse caso, pode-se observar que a ponderação embutida nesse indice se dá segundo o logaritmo do inverso de $p_{1}$ Podemos interpretar ET como uma medida de concentração, pois como nos ensinam Braga \& Mascolo (1982), temos que "considerando-se $p_{i}$ como a parcela de mercado da i-ésima firma, a entropia representaria o valor esperado do conteúdo informacional de uma mensagem que afirmasse que, tendo sido vendido um certo montante pela indústria em questão para um consumidor qualquer, a venda foi efetuada pela i-ésima firma. Desta forma, quanto maior a parcela de mercado de firma, menor o 'grau de surpresa' trazido pela mensagem, e vice-versa."

Desse modo, o indice de entropia de Theil pode ser considerado uma medida inversa de concentração.

O limite inferior desse indice, que corresponde a uma situação de concentração máxima, é igual a zero, pois nesse caso $p_{i}=1$ para algum i e $p_{j}=0$ para tódo $j \neq i$ (situação de monopólio).

o valor máximo do índice, correspondente a uma situação de concentração mínima, pode ser obtido pelo método de multiplicadores de l.agrange, onde está sendo utilizada a restrição de que as parcelas de mercado devem somar um.

$$
L=\sum_{i=1}^{n} p_{i}, \ln p_{i}-\lambda\left(\sum_{i=1}^{n} p_{i}-1\right)
$$

Igualando-se a derivada parcial de $\mathrm{L}, \mathrm{com}$ respeito a $\mathrm{p}_{\mathrm{b}}$, a zero, tem-se

$$
\frac{\partial L}{\partial p_{i}}=-1-\ln p_{i}-1=0
$$

Assim, obiém-se para $p_{i}$ o valor $e^{-\lambda-1}$, que substituido na restrição

$$
\sum_{i=1}^{n} p_{i}=1 \text { nos leva a } \lambda=\ln n-1
$$

Substituindo em (i) teromos

$$
-1-\ln p_{i}-\ln n+1=0
$$

logo $p_{i}=1 / n$, que utilizado na fórmula de $E T$, nos dá um valor máximo desse índice como sendo igual a $\ln n$, assim.

$$
0 \leq E T \leq \ln n
$$

Podemos estabelecer um número equivalente de firmas de igual tamanho, compativel com o indice ET observado. Uma igual participação entre firmas significa que $p_{i}=1 / n$. Substituindo esse valor na fórmula do indice de entropia temos:

$$
E T=\sum_{i=1}^{n} 1 / n \ln n=\ln n
$$


Logo o número equivalente de firmas $\left(n_{0}\right)$ é o antilogaritmo de $E T$ :

$$
n_{0}=e^{E T}
$$

Quando ET for mínimo $n_{0}=1$ e quando for máximo $n_{0}=n$, assim:

$$
1 \leq n_{0} \leq n
$$

Analogamente ao indice de Herfindahl, podemos definir uma versão do índice de entropia tal que a amplitude de variação se torne independente do número de observações. Tal medida, conhecida como entropia relativa (ER), consiste, portanto, na divisão do índice de entropia por In $n$. Assim procedendo tem-se que a amplitude de variação é constante e igual à unidade.

$$
\frac{\ln n}{\ln n}-\frac{0}{\ln n}=1
$$

Por outro lado, como o limite superior do indice de entropia (relativo a uma concentração mínima) coincide com a amplitude de variação, podemos interpretar a normalização proposta como expressando o grau de concorrência efetiva relativamente ao ideal. Os principais resultados referentes aos indices de Herfindahl e de entropia são resumidos no quadro a seguir.

Quadro 1 - Índices de concentração - principais características

\begin{tabular}{|l|c|c|c|c|}
\hline Fórmula & $\begin{array}{c}\text { Valor } \\
\text { minimo }\end{array}$ & $\begin{array}{c}\text { Valor } \\
\text { máximo }\end{array}$ & $\begin{array}{c}\text { Amplitude } \\
\text { de variação }\end{array}$ & $\begin{array}{c}\text { Sistema de } \\
\text { pesos das parcela } \\
\text { de mercado }\end{array}$ \\
\hline$H=\sum_{i=1}^{n} p_{i}^{2}$ & $1 / n$ & 1 & $(n-1) / n$ & $p_{i}$ \\
$H^{\prime}=\frac{n}{n-1} \sum_{i=1}^{n} p_{i}^{2}$ & $1 /(n-1)$ & $n /(n-1)$ & 1 & $p_{i}$ \\
$H^{\prime \prime}=\frac{1}{n-1}\left[n \sum_{i=1}^{n} p_{i}^{2}-1\right]$ & 0 & 1 & 1 & $p_{i}$ \\
$E T=\sum_{i=1}^{n} p_{i} \ln \left(1 / p_{i}\right)$ & 0 & $\ln n$ & $\ln n$ & $\ln \left(1 / p_{i}\right)$ \\
$E T=\frac{1}{\ln n} \sum_{i=1}^{n} p_{i} \ln \frac{1}{p_{i}}$ & 0 & 1 & 1 & $\ln \left(1 / p_{i}\right)$ \\
\hline
\end{tabular}

\section{ESCOLHA DE UMA MEDIDA DE CONCENTKAÇÃO ÓTIMA}

Toda a explanação anterior defende a superioridade da medida sumária de Herfindahl e de entropia em relação às populares razões de concentração. Existem, no entanto, autores como Bailey \& Boyle (1971) que apontam os citados índices como substitutos entre si. Para tanto, calcularam coeficientes de correlação entre os diversos índices tendo como base os dados da indústria americana, uma vez que constataram uma elevada correlação entre os diversos indices, sugeriram a 
utilização das razões de concentração dada a facilidade de cálculo. ${ }^{4}$ Todavia, como mostra Boyes \& Smyth (1979), uma perfeita correlação entre medidas de concentração alternativas não è suficiente para garantir a substitutibilidade entre essas, quando se deseja avaliar as mudanças na concentraçāo ao longo do tempo ou medir o grau de sensibilidade de uma variável à concentração (ou vice-versa). Vejamos a análise desenvolvida por tais autores para esse primeiro aspecto.

Suponha que a medida de concentração $C_{1}$ mude de $C_{1, t}$ no periodo t para $(1+g)$ $\mathrm{C}_{1, t}$ no período $\mathrm{t}+1$, ou seja:

(i)

$$
C_{t, t+1}=(1+g) C_{1, t}
$$

A pergunta relevante é: haverá um aumento correspondente na medida de concentração alternativa $C_{2}$ de $100 \mathrm{~g}$ por cento de $C_{2, t}$ em t para $(1+g) C_{2, t+1}$ em $t+1$ ? A resposta será afirmativa somente se $C_{1}$ e $C_{2}$ forem proporcionais. Suponha que $C_{1}$ e $\mathrm{C}_{2}$ sejam perfeitamente correlacionados e se situem ao longo da reta.

$$
c_{1}=a+b c_{2} \text { onde } b>0
$$

Admita que a relação (ii) seja válida para os periodos $t$ e $t+1$. Substituindo a expressão (ii) definida para o periodo $t+1$ em (i) e rearranjando tem-se:

$$
C_{2, t+1}=\frac{a g}{g}+(1+g) c_{2, t}
$$

Assim $C_{2}$ crescerá em $100 \mathrm{~g}$ por cento entre os períodos $t$ e $t+1$ se $a=0$, mas observando (ii) veremos que precisaremos de proporcionalidade entre os indices $\left(\mathrm{C}_{1}=\mathrm{bC}_{2}\right)$.

Podemos, contudo, estabelecer que não existe uma proporcionalidade entre as razöes de concentração e o índice de Herfindahl. De fato, fazendo uso da definição anteriormente apresentada, podemos expressar a razão de concentração das quatro maiores firmas em termos da participação média dessas firmas no mercado, assim temos:

$$
C R_{4}=4 \bar{p}_{4}
$$

Por cutro lado, sabemos que por definição o indice de Herfindahl é

$$
H=\sum p
$$

Agrupando as quatro maiores firmas e supondo que cada uma delas possui uma participação no mercado igual à média desse grupo, poderemos definir uma relaçāo entre $\mathrm{CR}_{4}$ e $\mathrm{H}$, teremos que:

Não temos, portanto, uma proporcionalidade entre $\mathrm{CR}_{4}$ e $\mathrm{H}, \mathrm{e}$

\footnotetext{
${ }^{4}$ No caso brasileiro, Braga \& Mascolo (1982) constataram coeficientes de correlação entre as razões de concentração e algumas medidas resumo, acima de 0.9 .

5 Para argumento relativos a razōes de concentração para $n>4$ veja Boyes e Smith (1979).
} 
conseqüentemente não se pode afirmar de forma simplista que essas medidas são substitutas entre si, em que pesem as hipóteses assumidas para se estabelecer uma relação entre esse dois índices.

Isso posto, a questão da escolha entre indices alternativos se coloca como fundamental, fazendo-se necessário o estabelecimento de propriedades desejáveis para um indice. Nesse sentido, Hall \& Tideman (1967) sugeriram as seguintes propriedades.

a) Deve ser uma medida unidimensional não ambigua, ou seja, um valor mais elevado do indice deve indicar uma estrutura mais concentrada;

b) deve ser independente do valor total da variável em estudo, sendo, pois, uma função das parcelas de mercado de todas firmas;

c) deve ser afetada por uma mudança em qualquer $p_{i}$, tal que mudanças na parcelas de mercado de firmas situadas em quaisquer posiçōes, em termos de um ranking, devem afetar a magnitude do indice;

d) se uma indústria $A$ tivesse $K$ vezes o número de firmas de uma indústria $B$, $e$ ainda se $K>1$ e as parcelas $p_{i}$ em $A$ são distribuídas tal que correspondendo a cada $p_{i}$ em $B$, existem $K$ firmas de tamanho $p_{i} / K$, então a medida de concentração relativa à $A$ deve ser $1 / K$ vezes a medida para $B$ Assim se cada firma fosse dividida em duas de tamanho idêntico, o indice deveria cair a metade. Tal propriedade ajudaria a configurar a cardinalidade da medida de concentração;

e) quando uma indústria está dividida em n firmas de tamanho idêntico, deve-se ter um decréscimo do valor da medida de concentraçäo, conforme n se eleva;

f) o índice deve variar entre 0 e 1 para facilidade de manipulação.

A inspeção dessas propriedades permite reenfatizar as deficiências das razões de concentração, já referidas anteriormente. De fato, esses indices só satisfazem as propriedades (a), (e) e (f), enquanto que o indice Herfindahl satisfaz todas elas. Cabe ressaltar, contudo, que um mesmo valor do indice Herfindahl é compativel com diversas distribuições de firmas, o que indica que destarte as propriedades acima serem importantes, qualquer indice que se considere terá sempre um caráter aproximado. De todo modo, a discussão anterior indica a utilização de medidas resumo, mormente em estudos de séries temporais, sempre que os dados estiverem disponiveis.

\section{BIBLICSRAFIA}

ADELMAN, M. A. The measurement of industrial concentration. Review of Economics anc Statistics, v.43, n.4, 196 ?

Comments on the ' $\mathrm{H}$ ' concentration measure as a number-equivalent. Review of Economics and Statistics, v. 51, n.1, 1969

BARBOSA, F. H. Medidas de Concentração. Revista de Econometria, v.1, n.1, 1981

BAILEY, D. \& BOYLE, S. E. The optimal measure of concentration. Journal of the American Statistical Society, v.66, 1971.

BAIN, J.S. Industrial organization. $2^{a}$ ed. New York: John Wiley \& Sons, 1968

BOYES, W. J. \& SMITH, D. F. The optimal concentration measure: theory an evidence for the canadian manufacturing industries. Applied Economics, v.11, n.3, 1979.

\footnotetext{
${ }^{6}$ Hall \& Tideman (1967) lembram que o índice de Herfindahl enfatiza os tamanhos relativos das firmas numa indústria. Segundo tais autores, há que se considerar também o tamanho absoluto das firmas; nesse sentido propõem uma medida de concentração alternativa que também satisfaz as seis propriedades tidas como desejáveis.
} 
BRAGA, H. C. \& MASCOLO, J. L. Mensuração da concentração industrial no Brasil. Pesquisa e Planejamento Econbmico, v.12, n.2, 1982.

FINKELSTEIN, M. O. \& FRIEBERG, R. M. The application of an entropy theory of concentration to the Clayton Act. Yale Law Joumal, 1967.

HALL, M. \& TIDEMAN, N. Measures of concentration. Journal of the American Statistical Association, v. 62, n. 317, 1967.

HERFINDAHL, O. C., Concentration in the steel industry. Tese de doutoramento, Columbia University, 1950.

SRIVASTAVA, D. K. \& AGGARWAL, P. K. Measuring tax revenue centralization in federal systems: a case stuy of India. Public Finance, v. 34, n.3, 1979.

THEIL. H. Economics and information theory. Amsterdam: North-Holland, 1967.

WEINSTOCK, L. Using the Herfindahl index to measure concentration, Antitrust Bulletin, v.27, n.2, 1982.

\section{ABSTRACT \\ MEASURES OF INDUSTRIAL CONCENTRATION: A SURVEY}

This paper aims at presenting some measures of industrial concentration, such as concentration reasons, Herfindahl index and Theil entropy index. Besides, it discusses the advantage of specific measures when they are related with others. 


\section{ERRATA}

Na edição de Análise Econômica de março/setembro de 1994 $\left(\mathrm{n}^{\text {os }} 21\right.$ e 22), cometemos algumas falhas de revisão nas fórmulas do artigo de Marcelo Resende, denominado Medidas de concentração industrial: uma resenha.

As correções são as seguintes:

a) p. 27, segunda fórmula: $\quad \partial L \partial p_{i}=2 p_{i}-\lambda=0$

(b) p. 29, terceira fórmula: $\quad \partial L \partial p_{i}=-1-\ln p_{i}-\lambda=0$

(c) p. 31, fórmula a ser acrescentada após a nota 5 :

$$
H=\left(4 \bar{p}_{4}\right)^{2}+\sum_{i=5}^{n} p_{l}^{2}=C R_{4}^{2}+\sum_{i=5}^{n} p_{l}^{2}
$$

